\title{
Research Communication \\ Genetic Analysis of MEFV Gene Pyrin Domain in Patients With Behçet's Disease
}

\author{
Ahmet Dursun, ${ }^{1}$ Hatice Gul Durakbasi-Dursun, ${ }^{2}$ Ayse Gul Zamani, ${ }^{2}$ Zerrin Gülin Gulbahar, ${ }^{3}$ \\ Recep Dursun, ${ }^{4}$ and Cengiz Yakicier ${ }^{3}$ \\ ${ }^{1}$ Department of Medical Genetics, Faculty of Medicine, Zonguldak Karaelmas University, 67600 Zonguldak, Turkey \\ ${ }^{2}$ Department of Medical Genetics, Meram Medical Faculty, Selcuk University, 42080 Konya, Turkey \\ ${ }^{3}$ Department of Molecular Biology and Genetics, Bilkent University, 06800 Ankara, Turkey \\ ${ }^{4}$ Department of Dermatology, Baskent University, 42080 Konya, Turkey
}

Received 3 January 2006; Revised 20 January 2006; Accepted 20 January 2006

\begin{abstract}
Objectives. Behçet's disease (BD) is a systemic vasculitis with recurrent oral and genital ulcers and uveitis. MEFV gene, which is the main factor in familial Mediterranean fever (FMF), is also reported to be a susceptibility gene for BD. The pyrin domain of MEFV gene is a member of death-domain superfamily and has been proposed to regulate inflammatory signaling in myeloid cells. This study was designed to determine if mutations in pyrin domain of MEFV gene are involved in BD. Methods. We analyzed the pyrin domain of MEFV gene in 54 Turkish patients with BD by PCR-analysis and direct sequencing. Results. Neither deletion or insertion mutations nor point mutations in pyrin domain were found in any patient. Conclusion. Although pyrin gene mutations have been reported in patients with $\mathrm{BD}$, pyrin domain is not mutated. However, alterations in other regions of MEFV gene and interaction between pyrin domains are needed to be further investigated.
\end{abstract}

Copyright (c) 2006 Ahmet Dursun et al. This is an open access article distributed under the Creative Commons Attribution License, which permits unrestricted use, distribution, and reproduction in any medium, provided the original work is properly cited.

\section{INTRODUCTION}

Behçet's disease (BD) is an unclassified systemic vasculitis with a chronic course. Although it was originally described with recurrent oral and genital ulcers and uveitis, it is now recognized as a multisystem disorder also affecting all types and sizes of blood vessels, the joints, the central nervous system, the lungs, and the intestines [1]. The pathogenesis of $\mathrm{BD}$ is not known, however, it possibly involves complex interactions of genetic and environmental factors. The manifestations of BD are considered to be developed as a result of immunological dysfunction, which is suggested to be induced by exogenic pathogens in genetically susceptible individuals and includes hyperreactivity of neutrophils, overexpression of several proinflammatory and Th1-type cytokines, and several phenotypic and functional lymphocyte abnormalities [2,3].

$M E F V$ gene mutations have been reported to be responsible for familial Mediterranean fever (FMF) which is an autosomal recessive and is also known as an autoinflammatory disease that is characterized by recurrent episodes of unseemingly unprovoked inflammation that, unlike autoimmune disorders, lack the production of high-titer autoantibodies or antigen-specific $\mathrm{T}$ cells [4]. MEFV gene has also been suggested recently to be a susceptibility gene for $\mathrm{BD}[5,6]$. MEFV gene, was identified in 1997 by positional cloning [7] encoding a 781-amino-acid protein, pyrin, which is predominantly expressed in polymorphonuclear leukocytes (PMNs) and cytokine-activated monocytes [8]. Pyrin consists of four functional domains, a B-box zinc-finger domain, a coiledcoil domain, a C-terminal B30.2 domain, and a 92-aminoacid $\mathrm{N}$-terminal pyrin domain that is shared by a number of other proteins involved in apoptosis and inflammation [9].

The pyrin domain is a member of the six-helix bundle, death-domain superfamily that includes death domains, death effector domains, and caspase recruitment domains (CARDs) [10]. Although the function of pyrin protein remains to be determined, it has been proposed to regulate inflammatory signaling in myeloid cells [11]. It has been suggested that pyrin domain, as a novel protein module, is found in proteins that are thought to function in apoptotic and inflammatory signaling pathways [11]. BD is not a Mendelian disorder; however, considering its occasional familial presentation and its close association with genes of major histocompatibility complexes, $\mathrm{BD}$ is under some sort of genetic control [12]. As MEFV gene mutations were present in $\mathrm{BD}$, this 
study was designed to determine whether mutations of pyrin domain of MEFV gene are related to $\mathrm{BD}$ and its inflammatory process.

\section{MATERIALS AND METHODS}

A total of 54 Turkish patients with Behçet's disease were included in this study. Patients with Behçet's disease were all fulfilling at least three of the International Study Group [13] criteria for BD and were clinically and serologically diagnosed by Department of Dermatology, Meram Medical Faculty, Selcuk University, 29 out of 54 patients were females.

\section{$P C R$, sequencing, and mutational analysis}

Specific primers for PCR amplification (406 bp) and sequencing of MEFV gene pyrin domain were designed using the Primer 3 program (PF: 5'-CAACCTGCCTTTTCTTGCTC-3', PR 5'-CACTCAGCACTGGATGAGGA$3^{\prime}$ ) (http://www.genome.wi.mit.edu/cgibin/primer/primer3 _www.cgi). Genomic DNA from peripheral blood cells was extracted using the QIAamp Blood Kit according to the manufacturer's instructions. PCR reaction was carried out in $50 \mu \mathrm{L}$ of solution containing $100 \mathrm{ng}$ of genomic DNA, $0.5 \mu \mathrm{mol} / \mathrm{L}$ of each primer, $200 \mu \mathrm{mol} / \mathrm{L}$ of each dNTP, $20 \mathrm{mmol} / \mathrm{L}$ of TrisHCl (pH 8.5), $50 \mathrm{mmol} / \mathrm{L}$ of $\mathrm{KCl}$, $3 \mathrm{mmol} / \mathrm{L}$ of $\mathrm{MgCl}_{2}$, and $1.0 \mathrm{U}$ of Taq polymerase (Qiagen). The amplification was performed on thermocycler (Perkin Elmer 9600), with a predenaturing procedure for 4 minutes at $94^{\circ} \mathrm{C}$ for 35 cycles (denaturing at $94^{\circ} \mathrm{C}$ for 1 minute, annealing at $60^{\circ} \mathrm{C}$ for 1 minute, and extension at $72^{\circ} \mathrm{C}$ for 1 minute), followed by an additional 10 -minute incubation at $72^{\circ} \mathrm{C}$. PCR products were purified with QIAquick PCR Purification Kit (QIAGEN) and sequencing was performed by using Amersham Dynamic ET Terminator Cycle Sequencing Kit and Perkin Elmer Big Dye Terminator Kit versus 3.1 with F\&R primers in both directions and analyzed in ABI 310 sequencer. The MEFV first exon sequences were aligned and analyzed using Mutation Explorer (DEMO) version 2.41 software (Softgenetics Inc).

\section{RESULTS}

We have carried out MEFV pyrin domain mutational analysis on 54 Turkish patients with Behçet's disease. A unique 406 bp fragment successfully amplified by PCR for all 54 samples was tested. This suggests that there were no detectable genomic deletions or insertions concerning pyrin domain of MEFV gene (Figure 1). Same PCR products were purified and used for direct sequencing to analyze single nucleotide changes. These 54 samples were successfully sequenced and no mutations in pyrin domain coding sequence and its immediately flanking sequences were observed (Figure 2).

\section{DISCUSSION}

Modular protein-protein interaction domains play an important role in many intracellular signal transduction path- ways [14]. In inflammation and apoptosis signaling pathways, three major families of protein modules have been proposed: the death domain (DD), the death effector domain (DED), and the caspase recruitment domain (CARD) [15]. These protein modules of approximately 100 amino acids in length function to mediate homotypic protein-protein interaction between signaling components leading to the activation of specific downstream targets. They are all from $\alpha$-helical bundles acting as adapters in signaling pathways and recruiting other proteins into signaling complexes [16]. These domains are required for the transmission and regulation of signals from receptor to effector, such as caspases, via homotypic interactions in which DDs interact with DDs, DEDs interact with DEDs, and CARDs interact with CARDs [17]. Moreover, despite their low degree of sequence similarity, these homotypic interaction domains have been proved to share a common three-dimensional fold, classified as death-domain fold [18].

At the time of the FMF susceptibility gene discovery, the function of pyrin was unknown, however, a significant breakthrough occurred when pyrin was found to be a member of the death fold superfamily $[11,19]$, with its $\mathrm{N}$ terminal pyrin domain (PYD) homologous to the death domain (DD), death effector domain (DED), and caspase recruitment domain (CARD) subfamilies. Pyrin protein has an important role in both NF-kB transcription factor activation and apoptosis, however, the exact details have not been sufficiently predictive. There is evidence that pyrin inhibits both NF-kB activation and apoptosis, induced by the ASC (apoptosis-associated speck-like protein containing a CARD) adaptor protein, by disruption of the interaction between ASC and caspase-8 [20].

Pyrin deficient mice have a defect in apoptosis, suggesting a proapoptotic role for pyrin through caspase recruitment, and full-length pyrin competes in vitro with caspase-1 for binding to ASC, a known caspase-1 activator, thus inhibiting pro-IL-1b cytokine processing to the active form [21].

Further insights into the function of pyrin have recently been revealed by an unusual collusion of two experiments of nature. Pyogenic arthritis, pyoderma gangrenosum, and acne (PAPA) syndrome are characterized by polymorphonuclear leukocyte invasion of joints and skin, producing a destructive arthritis and skin lesions, which can be extensive and disfiguring in some cases. This disease is due to mutations in the adaptor protein, praline serine threonine phosphatase-interacting protein 1 (PSTPIP1), which is a tyrosine-phosphorylated protein involved in cytoskeletal organization; these mutations result in altered binding of PSTPIP1 to the PEST-type protein tyrosine phosphatase (PTPPEST, AAA36529) [22]. In a search for pyrin binding proteins, Shoham et al studied cells from patients with PAPA syndrome, and, by using a combination of techniques including yeast two-hybrid assay: coimmunoprecipitation and coimmunofluorescence, have demonstrated an interaction between pyrin and PSTPIP1 [23]. They have thus revealed a biochemical pathway common to both FMF and PAPA syndromes. 


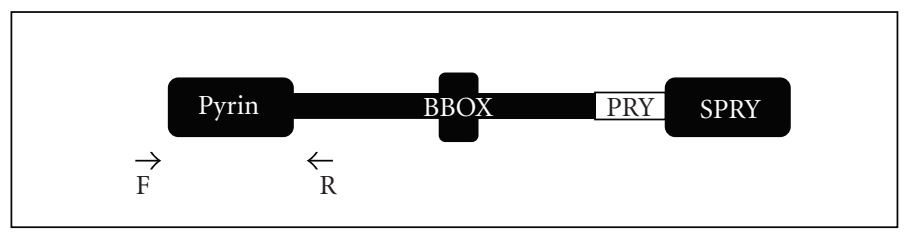

FIGURE 1: Diagram depicting the conserved domains of the human MEFV protein. Arrows indicate genomic localization of primers used for pyrin domain amplification.

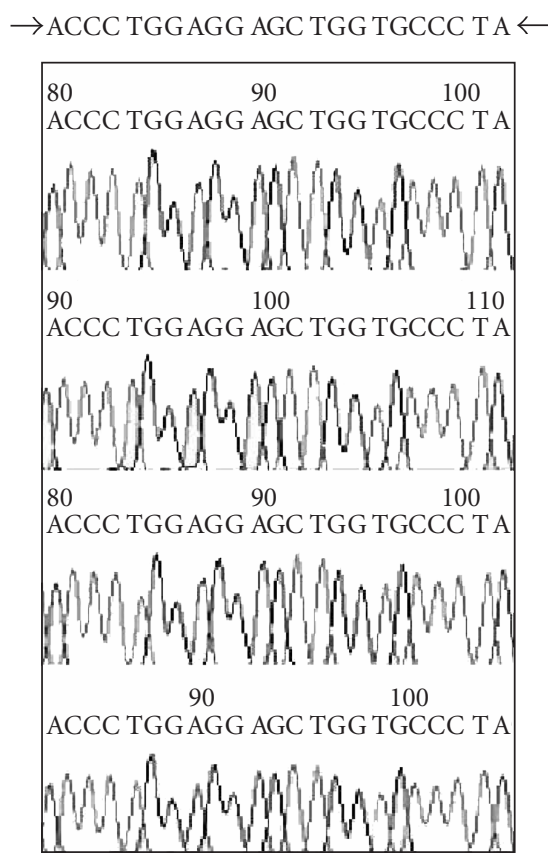

FIGURE 2: Representative results from 4 patients' direct DNA sequencing of PCR product for MEFV gene pyrin domain and wild type sequence of presented region.

PYRIN domain is also present in apoptosis-associated speck-like protein (ASC) and target of methylation-induced silencing 1 (TMS1), which functions as a positive mediator of apoptosis $[11,24]$. Inflammation and apoptosis upregulate ASC in neutrophils and, depending on the cellular context, it can either inhibit or activate NF-kB [25]. ASC contains both a pyrin and a CARD domain. ASC and pyrin seem to interact via their pyrin domains, while the CARD domain of ASC was shown to bind to the CARD domain of caspase-1 $[24,26]$. The pyrin domain of ASC was further shown to bind to POP1/ASC2, a small protein consisting of a single pyrin domain with a high level of amino-acid-sequence similarity to the pyrin domain of ASC [27]. The interaction between ASC and POP1/ASC2 results in a modulation of NF-kB and procaspase-1 regulation [27]. Finally, there is evidence that ASC and caspase-1, together with NALP1 (another PYRINdomain protein) and caspase-5, form a proapoptotic complex, named inflammasome, which is essential for innate immunity involving LPS-induced apoptosis [28].
Apoptotic and inflammatory roles of the pyrin were further demonstrated via caspase activation. The pyrin domain has a role as adaptor between NALP3 (receptor) and caspase 1 (effector) in NALP3 inflammasome production. This leads to the cytokine activation and apoptosis [29].

Two further human hereditary diseases were recently attributed to the pyrin-domain protein: Muckle-Wells syndrome and familial cold autoinflammatory syndrome [30].

Although pyrin domains occur in more than 20 human proteins, only a few additional pyrin-domain proteins have been characterized functionally. Almost all of them appear to be involved in apoptosis and inflammation [31, 32].

There have been reports that pyrin mutations might not be enough for clinical outcome for PAPA syndrome. Although pyrin regulates the IL-1b pathway, it also influences $\mathrm{NF}-\mathrm{kB}$ activation and apoptosis, and therefore, even if the interaction with pyrin is the most important one in PAPA, there might be other pyrin-dependent effects apart from the regulation of IL-1 b activation [22].

To the best of our knowledge, no mutation analysis is available for pyrin domain of the MEFV gene. Considering that pyrin-domain proteins interact frequently with other pyrin-domain proteins, pyrin-pyrin interactions are likely an important feature of pyrin-domain function. In conclusion, we have presented the results of pyrin-domain mutation screening from $54 \mathrm{BD}$ patients. Although, MEFV gene mutations have been reported as the cause of $\mathrm{BD}$, pyrin domain has not been mutated. On the other hand, our results do not exclude the possibility that the MEFV gene is inactivated by mutations located regions other than pyrin domain or another molecular mechanism. Interaction between pyrin domains needs to be further investigated, thus, pathophysiological mechanisms of autoinflammatory diseases might be explained.

\section{REFERENCES}

[1] T Sakane, M Takeno, N Suzuki, and G Inaba. Behçet's disease. The New England Journal of Medicine. 1999;341(17):12841291.

[2] A Gul. Behçet's disease: an update on the pathogenesis. Clinical and Experimental Rheumatology. 2001;19(5 suppl 24):S6S12.

[3] M Zierhut, N Mizuki, S Ohno, et al. Immunology and functional genomics of Behçet's disease. Cellular and Molecular Life Sciences. 2003;60(9):1903-1922.

[4] I Aksentijevich, M Centola, Z-M Deng, et al. Ancient missense mutations in a new member of the RoRet gene family are likely 
to cause familial Mediterranean fever. The International FMF Consortium. Cell. 1997;90(4):797-807.

[5] P Atagunduz, T Ergun, and H Direskeneli. MEFV mutations are increased in Behçet's disease (BD) and are associated with vascular involvement. Clinical and Experimental Rheumatology. 2003;21(4 suppl 30):S35-S37.

[6] N Imirzalioglu, A Dursun, B Tastan, Y Soysal, and M C Yakicier. MEFV gene is a probable susceptibility gene for Behçet's disease. Scandinavian Journal of Rheumatology. 2005;34(1):5658.

[7] The French FMF Consortium. A candidate gene for familial Mediterranean fever. Nature Genetics. 1997;17(1):25-31.

[8] M Centola, G Wood, D M Frucht, et al. The gene for familial Mediterranean fever, MEFV, is expressed in early leukocyte development and is regulated in response to inflammatory mediators. Blood. 2000;95(10):3223-3231.

[9] E Mansfield, J J Chae, H D Komarow, et al. The familial Mediterranean fever protein, pyrin, associates with microtubules and colocalizes with actin filaments. Blood. 2001; 98(3):851-859.

[10] D L Kastner and J J O'Shea. A fever gene comes in from the cold. Nature Genetics. 2001;29(3):241-242.

[11] J Bertin and P S DiStefano. The PYRIN domain: a novel motif found in apoptosis and inflammation proteins. Cell Death and Differentiation. 2000;7(12):1273-1274.

[12] T Chajek and M Fainaru. Behçet's disease report of 41 cases and a review of the literature. Medicine. 1975;54(3):179-195.

[13] The International Study Group for Behçet's disease. Evaluation of diagnostic ('classification') criteria in Behçet's diseasetowards internationally agreed criteria. British Journal of Rheumatology. 1992;31(5):299-308.

[14] T Pawson and P Nash. Protein-protein interactions define specificity in signal transduction. Genes \& Development. 2000;14(9):1027-1047.

[15] K Hofmann. The modular nature of apoptotic signaling proteins. Cellular and Molecular Life Sciences. 1999;55(8-9):11131128.

[16] C H Weber and C Vincenz. The death domain superfamily: a tale of two interfaces? Trends in Biochemical Sciences. 2001; 26(8):475-481.

[17] E Liepinsh, R Barbals, E Dahl, A Sharipo, E Staub, and G Otting. The death-domain fold of the ASC PYRIN domain, presenting a basis for PYRIN/PYRIN recognition. Journal of Molecular Biology. 2003;332(5):1155-1163.

[18] A G Murzin, S E Brenner, T Hubbard, and C Chothia. SCOP: a structural classification of proteins database for the investigation of sequences and structures. Journal of Molecular Biology. 1995;247(4):536-540.

[19] F Martinon, K Hofmann, and J TschoThe PYRIN domain: a possible member of the death domain-fold family implicated in apoptosis and inflammation. Current Biology. 2001;11(4):R118-R120.

[20] J Masumoto, T A Dowds, P Schaner, et al. ASC is an activating adaptor for NF-kappa B and caspase-8-dependent apoptosis. Biochemical and Biophysical Research Communications. 2003;303(1):69-73.

[21] J J Chae, H D Komarow, J Cheng, et al. Targeted disruption of pyrin, the FMF protein, causes heightened sensitivity to endotoxin and a defect in macrophage apoptosis. Molecular Cell. 2003;11(3):591-604.

[22] C A Wise, J D Gillum, C E Seidman, et al. Mutations in CD2BP1 disrupt binding to PTP PEST and are responsible for PAPA syndrome, an autoinflammatory disorder. Human Molecular Genetics. 2002;11(8):961-969.
[23] N G Shoham, M Centola, E Mansfield, et al. Pyrin binds the PSTPIP1/CD2BP1 protein, defining familial Mediterranean fever and PAPA syndrome as disorders in the same pathway. Proceedings of the National Academy of Sciences of the United States of America. 2003;100(23):13501-13506.

[24] N Richards, P Schaner, A Diaz, et al. Interaction between PYRIN and the apoptotic speck protein (ASC) modulates ASC-induced apoptosis. The Journal of Biological Chemistry. 2001;276(42):39320-39329.

[25] M Shiohara, S Taniguchi, J Masumoto, et al. ASC, which is composed of a PYD and a CARD, is up-regulated by inflammation and apoptosis in human neutrophils. Biochemical and Biophysical Research Communications. 2002;293(5):13141318.

[26] J Masumoto, S Taniguchi, J Nakayama, et al. Expression of apoptosis-associated speck-like protein containing a caspase recruitment domain, a PYRIN N-terminal homology domaincontaining protein, in normal human tissues. The Journal of Histochemistry and Cytochemistry. 2001;49(10):1269-1275.

[27] C Stehlik, M Krajewska, K Welsh, S Krajewski, A Godzik, and J C Reed. The PAAD/PYRIN-only protein POP1/ASC2 is a modulator of ASC-mediated nuclear-factor- $\kappa$ B and procaspase-1 regulation. The Biochemical Journal. 2003;373(pt 1):101-113.

[28] F Martinon, K Burns, and J TschoThe inflammasome: a molecular platform triggering activation of inflammatory caspases and processing of proIL- $\beta$. Molecular Cell. 2002;10(2): $417-426$.

[29] F Martinon and J TschoInflammatory caspases: linking an intracellular innate immune system to autoinflammatory diseases. Cell. 2004;117(5):561-574.

[30] H M Hoffman, J L Mueller, D H Broide, A A Wanderer, and R D Kolodner. Mutation of a new gene encoding a putative PYRIN-like protein causes familial cold autoinflammatory syndrome and Muckle-Wells syndrome. Nature Genetics. 2001; 29(3):301-305.

[31] L Fiorentino, C Stehlik, V Oliveira, M E Ariza, A Godzik, and J C Reed. A novel PAAD-containing protein that modulates NF- $\kappa$ B induction by cytokines tumor necrosis factor- $\alpha$ and interleukin-1 $\beta$. The Journal of Biological Chemistry. 2002; 277(38):35333-35340.

[32] J A Harton, M W Linhoff, J Zhang, and J P Ting. Cutting edge: CATERPILLER: a large family of mammalian genes containing CARD, PYRIN, nucleotide-binding, and leucine-rich repeat domains. Journal of Immunology. 2002;169(8):4088-4093. 


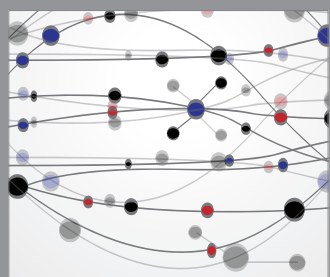

The Scientific World Journal
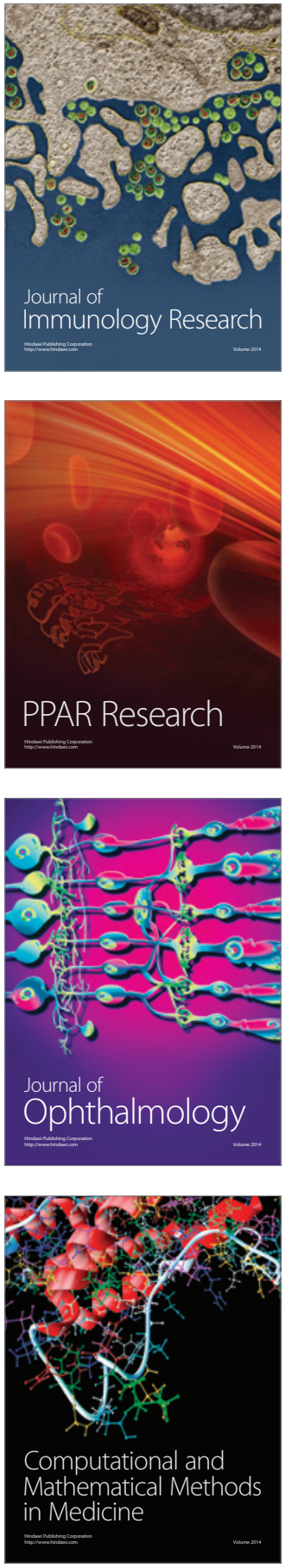

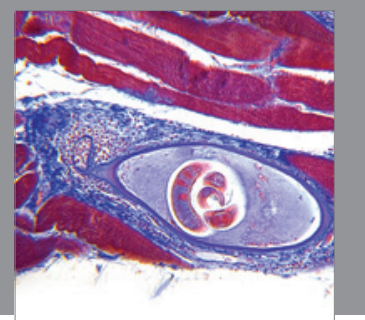

Gastroenterology

Research and Practice
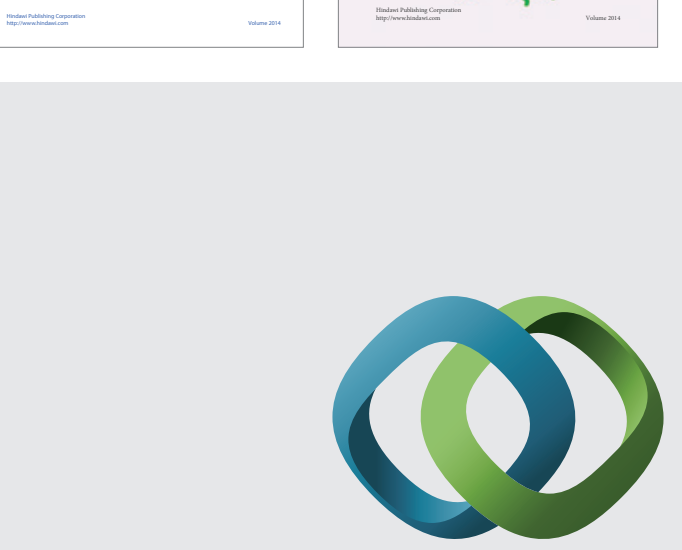

\section{Hindawi}

Submit your manuscripts at

http://www.hindawi.com
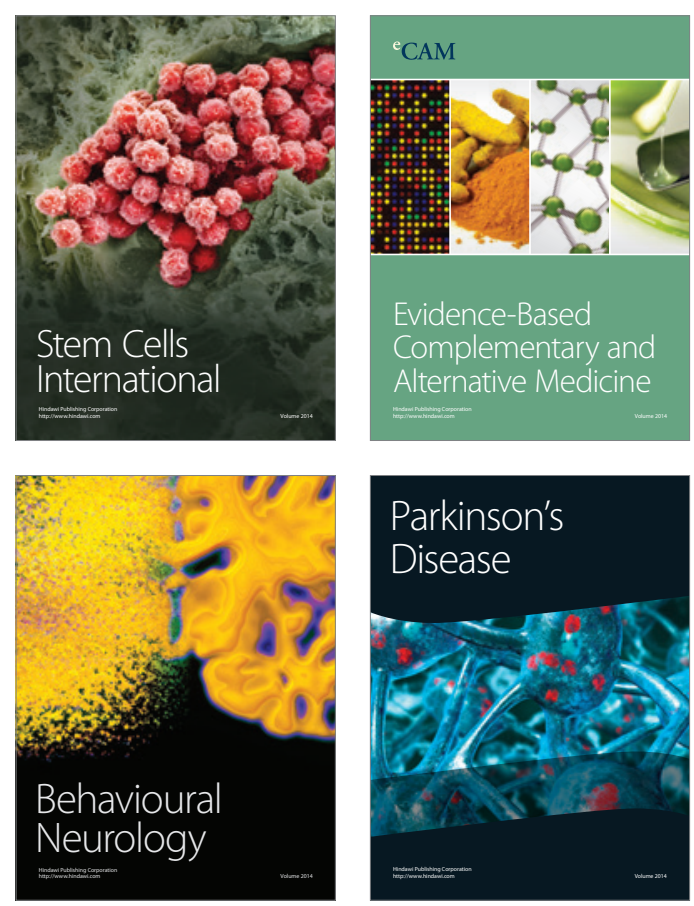

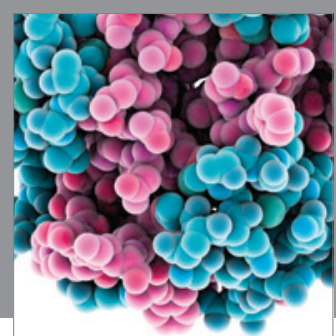

Journal of
Diabetes Research

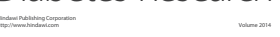

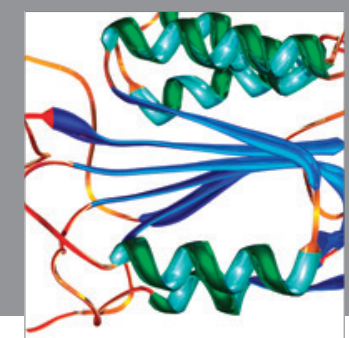

Disease Markers
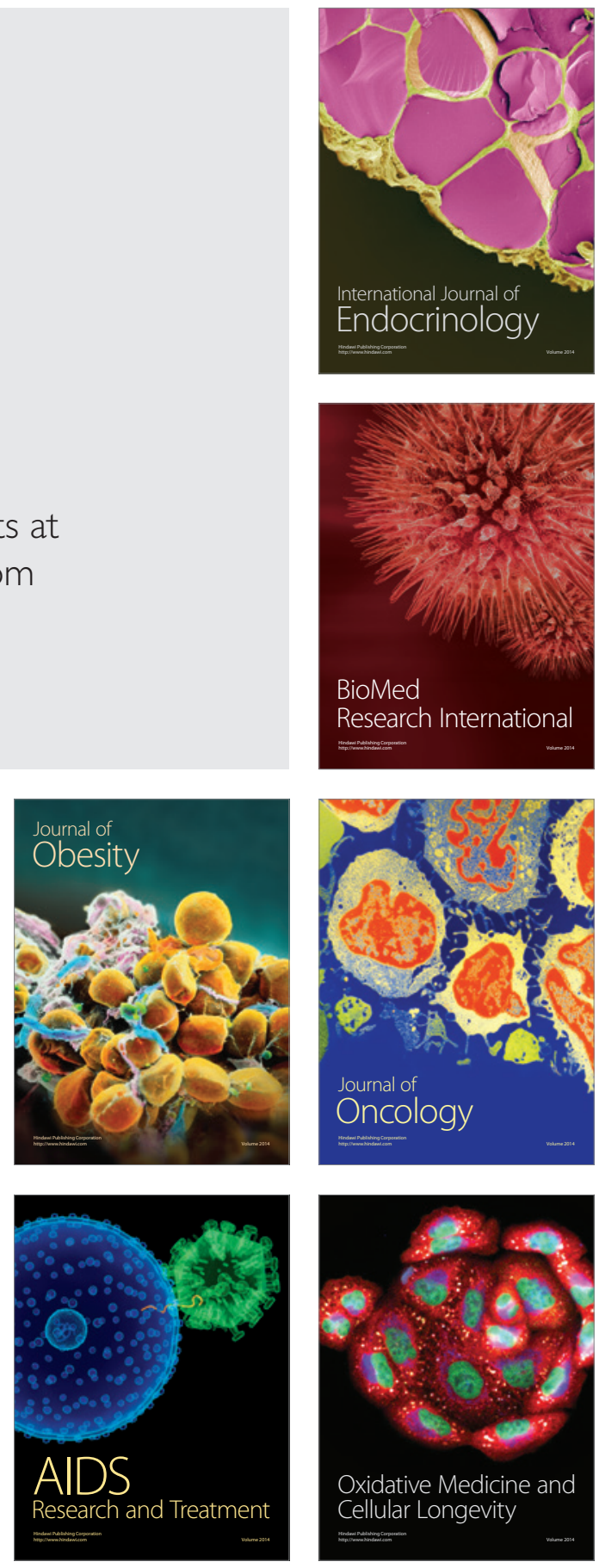